\title{
Tumor Growth Simulation Profiling
}

\author{
Claire Jean-Quartier ${ }^{(凶)}$, Fleur Jeanquartier, David Cemernek, \\ and Andreas Holzinger
}

Holzinger Group, Research Unit HCI-KDD, Institute for Medical Informatics, Statistics and Documentation, Medical Informatics, Statistics and Documentation, Medical University of Graz, Graz, Austria

c. jeanquartier@hci-kdd.org

\begin{abstract}
Cancer constitutes a condition and is referred to a group of numerous different diseases, that are characterized by uncontrolled cell growth. Tumors, in the broader sense, are described by abnormal cell growth and are not exclusively cancerous. The molecular basis involves a process of multiple steps and underlying signaling pathways, building up a complex biological framework. Cancer research is based on both disciplines of quantitative and life sciences which can be connected through Bioinformatics and Systems Biology. Our study aims to provide an enhanced computational model on tumor growth towards a comprehensive simulation of miscellaneous types of neoplasms. We create model profiles by considering data from selected types of tumors. Growth parameters are evaluated for integration and compared to the different disease examples.

Herein, we describe an extension to the recently presented visualization tool for tumor growth. The integration of profiles offers exemplary simulations on different types of tumors. The enhanced biocomputational simulation provides an approach to predicting tumor growth towards personalized medicine.
\end{abstract}

Keywords: Computational biology - Cancer types - Tumor growth • Simulation · HCI · Visualization · Systems biology · Kinetics · Data visualization

\section{Introduction}

Tumors or neoplasms constitute an overgrowth of tissue, often called mass and lump. Tumors can be non-cancerous (benign) or become cancerous (malignant).

The biological background involves a multistep development based on genomic instability, metabolic reprogramming and evasion from the immune system $[1,2]$. Underlying signaling events comprise proliferation and growth promotion, cell death inhibition and replicative immortality, neoangiogenesis and invasion [3].

Classification and prediction of the different types of cancer is crucial for therapy and treatment strategies against the often deadly medical condition. Over 
the last years, diagnostic classification techniques have made progress advancing from microscopic examination of morphological tissue changes towards gene analysis and biomarker discoveries $[4,5]$. Respective analysis methods are known for the necessity of computerized support including statistical methods and machine learning classifiers [6]. Yet, methods for predicting tumor growth and cancer prognosis have not fully advanced from basic research to clinical investigations [7]. Still, mathematical and computational models for cancer prediction function as beneficial resource in cancer research. There are several models each on individual types of cancer including brain, ovarian, colon cancers, melanoma, leukemia or head/neck tumors [9]. These models range from deterministic to stochastic, from continuous population dynamics to agent-based individual cell models, from fluid dynamics to Monte Carlo simulations or energy minimization models and are differentially used to describe the various phases of initiation, growth, invasion and migration $[10,11]$. Moreover, so-called hybrid models simulate variable discrete and continuous aspects on intracellular and intercellular processes [12].

Enhanced modeling tools can provide indications and quantitative criteria for the prognosis of tumor progression as well as therapeutic strategies and support decision making $[7,8]$.

\section{Approach}

Our study is based on the recently presented simulation on tumor growth [13], which is available at Github: https://github.com/davcem/cpm-cytoscape and as Online Demo: http://styx.cgv.tugraz.at:8080/cpm-cytoscape/. Figure 1 shows a view from the web-tool for tumor growth modeling and its graphical output. The tool depicts an biocomputational approach to simulate biological cell sorting using a two-dimensional extended Potts model $[14,15]$ for the use case of tumor growth on the cellular level.

By scanning through literature and web resources, we consider data from selected types of tumors. These are scanned for usable growth parameters. We create model profiles of exemplary pre-settings for an integration into the simulation. The profiles are compared and evaluated to the different disease examples.

In regard to ease of use for medical scientists, we further improve the graphical user interface for choosing between the model profiles.

\section{Results \& Discussion}

We choose three distinct types of cancer and integrated predefined settings based on recently presented studies on ovarian cancer [16], colorectal cancer [17] and brain cancer [18].

Figure 3 demonstrates the option for choosing between the model profiles for use cases of various types of cancer (Fig. 2).

Table 1 summarizes the selected parameters for the different profiles. Examples are given as custom build as well as ovarian, colorectal and brain cancer 


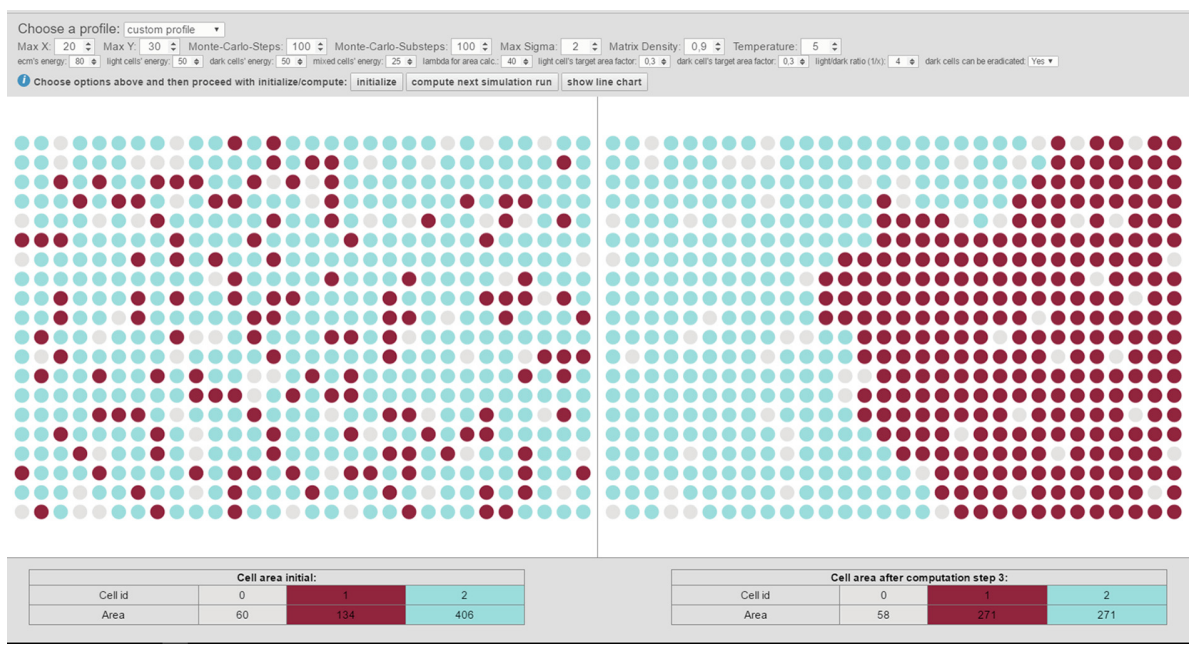

Fig. 1. Screenshot of the tool's graphical view

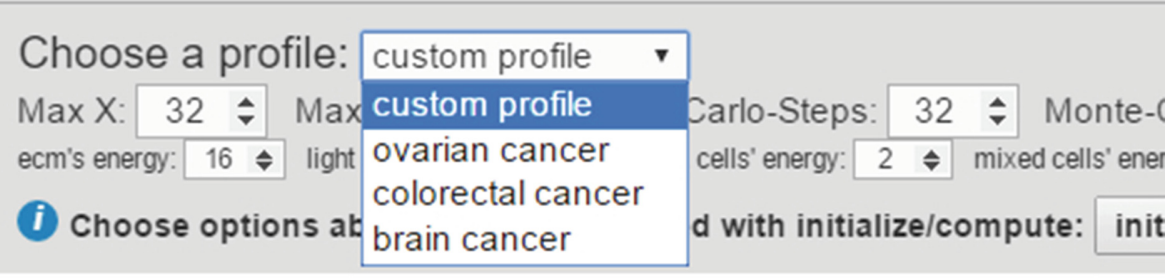

Fig. 2. Screenshot of profile selection via the tool's user interface

Table 1. CPM parameter settings: comparison of settings for custom and profile builds in case of two exemplary cell types corresponding to three different cancer diseases (ovarian, colorectal and brain, adopted from [16-18]).

\begin{tabular}{|c|c|c|c|c|c|c|c|c|c|}
\hline & \multicolumn{2}{|c|}{$\operatorname{Max} \mathbf{X} * \mathbf{Y}$} & \multicolumn{3}{|c|}{ MCS, substeps } & $\operatorname{Max} \sigma$ & \multicolumn{2}{|c|}{ Matrix density } & $\mathbf{T}$ \\
\hline Custom & \multicolumn{2}{|c|}{$32 * 32$} & \multicolumn{3}{|c|}{32,64} & 2 & \multicolumn{2}{|c|}{0.8} & 10 \\
\hline Ovarian cancer & \multicolumn{2}{|c|}{$162 * 50$} & \multicolumn{3}{|c|}{10,135} & 2 & \multicolumn{2}{|c|}{1} & 10 \\
\hline Colorectal cancer & \multicolumn{2}{|c|}{$50 * 110$} & \multicolumn{3}{|c|}{55,10} & 2 & \multicolumn{2}{|c|}{0.91} & 0.1 \\
\hline \multirow[t]{2}{*}{ Brain cancer } & \multicolumn{2}{|c|}{$50 * 50$} & \multicolumn{3}{|c|}{250,10} & 2 & \multirow{2}{*}{\multicolumn{3}{|c|}{\begin{tabular}{c|c|c}
1 & 4 \\
$k)$ & Ratio $_{\text {light/dark }}$
\end{tabular}}} \\
\hline & 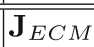 & 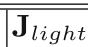 & $\overline{\mathbf{J}_{\text {dark }}}$ & 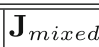 & $\overline{\lambda \lambda}$ & $\overline{\mathbf{A}_{t(l i g h t)}}$ & & & \\
\hline Custom & 16 & 15 & 2 & 11 & 0.05 & 0.4 & 0.4 & $1 /$ & \\
\hline Ovarian cancer & 5 & -15 & -10 & -1 & 3 & 0.0086 & 0.0089 & $1 /$ & \\
\hline Colorectal cancer & 0.2 & 0.1 & 0.1 & 0.2 & 0.1 & 0.0046 & 0.018 & $1 /$ & \\
\hline Brain cancer & 8 & 30 & 3 & 2 & 1 & 1 & 1 & $1 /$ & \\
\hline
\end{tabular}




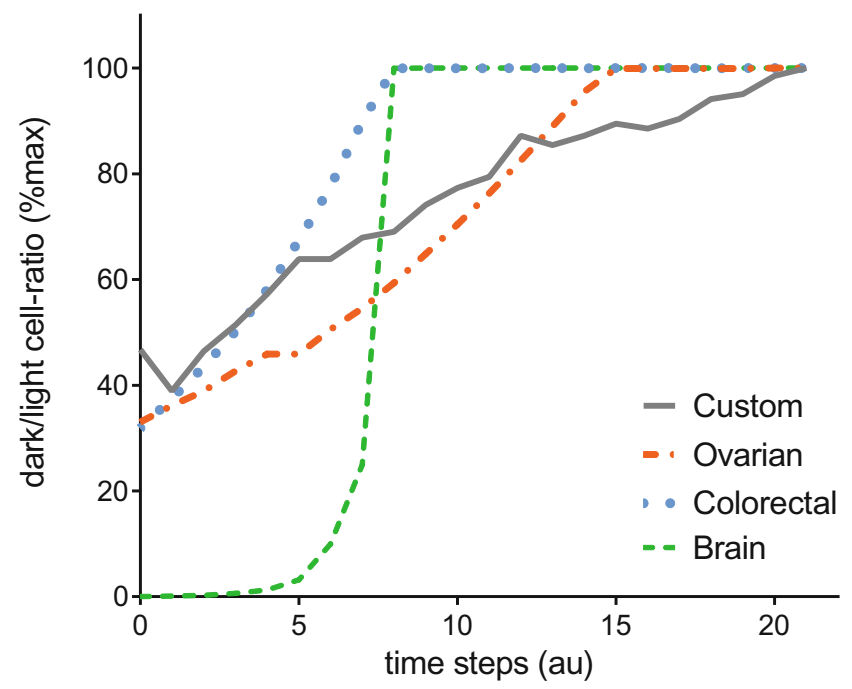

Fig. 3. Cell growth corresponding to various profiles: line chart showing representative ratios between numbers of dark and light cells, given as \% max, over computed steps as time.

[13,16-18]. The underlying factors have been adapted in order to be comparable within the tool's framework. While testing identified growth parameters, a key challenge is providing the possibility of dynamic adjustments of cell-related parameters to meet the needs for handling peculiarities of the various use cases of cancer diseases.

The integrated profiles depict distinct characteristics. The three selected examples are compared to the default settings from the custom profile. Representative growth rates differ in kinetic characteristics in terms of relative average and instantaneous growth rate as well as aggressivity and mortality. Figure 3 summarizes ratios between numbers of dark and light cells over time steps, calculated for the different profiles. The use case for brain cancer exhibits the highest growth rate of all selected cases and correlates to exponential growth succeeded by a sudden saturation, which can be translated as the spatial limitation within a biological compartment.

The simplified examples represent basic profiles suitable for likewise human or animal models. Parameters have to be further refined in order to reproduce realistic scenarios for more detailed studies on various aspects of tumor growth, in particular referring to the various cancer diseases.

Due to the variability within cancer classes, refined models are of main interest to supporting the evaluation of tumor aggressiveness and risk assessment. 
They provide a resource to study the inter- as well as intracellular system in correlation to temporal and spatial dynamics.

\section{Conclusion}

We believe that our approach offers the possibility to study tumor growth towards a comprehensive interpretation of tumor dynamics. Herein, we present simulation examples for studying multiple cancer diseases in regard to comparison of kinetic characteristics towards prediction. Results support the notion that tumor growth follows a universal law at first sight but has to be refined and reevaluated in detail.

\section{References}

1. Drake, J.W., Charlesworth, B., Charlesworth, D., Crow, J.F.: Rates of spontaneous mutation. Genetics 148(4), 1667-1686 (1998)

2. Lodish, H., Berk, A., Zipursky, S.L., et al.: Molecular Cell Biology, 4th edn. W. H. Freeman, New York (2000)

3. Hanahan, D., Weinberg, R.A.: Hallmarks of cancer. Cell 674(5), 646-646 (2011)

4. Cortés, J., et al.: New approach to cancer therapy based on a molecularly defined cancer classification. CA: Cancer J. Clin. 64(1), 70-74 (2014)

5. Rodríguez-Enríquez, S., Pacheco-Velázquez, S.C., Gallardo-Pérez, J.C., MarnHernández, A., Aguilar-Ponce, J.L., Ruiz-García, E., Ruizgodoy-Rivera, L.M., Meneses-García, A., Moreno-Sánchez, R.: Multi-biomarker pattern for tumor identification and prognosis. J. Cell Biochem. 112(10), 2703-15 (2011)

6. Wang, Y., Tetko, I.V., Hall, M.A., Frank, E., Facius, A., Mayer, K.F., Mewes, H.W.: Gene selection from microarray data for cancer classification-a machine learning approach. Comput. Biol Chem. 29(1), 37-46 (2005)

7. Vickers, A.J.: Prediction models in cancer care. CA: Cancer J. Clin. 61(5), 315-326 (2011). doi:10.3322/caac. 20118

8. Li, X.L., Oduola, W.O., Qian, L., Dougherty, E.R.: Integrating multiscale modeling with drug effects for cancer treatment. Cancer Inform. 14(Suppl. 5), 21-31 (2016)

9. Enderling, H., Rejniak, K.A.: Simulating cancer: computational models in oncology. Front Oncol. 3, 233 (2013)

10. Edelman, L.B., Eddy, J.A., Price, N.D.: In silico models of cancer. Wiley Interdisc. Rev. Syst. Biol. Med. 2(4), 438-459 (2010)

11. Benzekry, S., Lamont, C., Beheshti, A., Tracz, A., Ebos, J.M., Hlatky, L., Hahnfeldt, P.: Classical mathematical models for description and prediction of experimental tumor growth. PLoS Comput. Biol. 10(8), e1003800 (2014)

12. Rejniak, K.A., Anderson, A.R.A.: Hybrid models of tumor growth. Wiley Interdiscip Rev. Syst. Biol. Med. 3(1), 115-125 (2011)

13. Jeanquartier, F., Jean-Quartier, C., Cemernek, D., Holzinger, A.: In Silico Modeling For Tumor Growth Visualization. - Manuscript in revision (2016). https:// github.com/davcem/cpm-cytoscape/

14. Graner, F., Glazier, J.A.: Simulation of biological cell sorting using a twodimensional extended Potts model. Phys. Rev. Lett. 69, 2013-2016 (1992)

15. Szab, A., Merks, R.M.: Cellular potts modeling of tumor growth, tumor invasion, and tumor evolution. Frontiers in oncology 3 (2013) 
16. Giverso, C., Scianna, M., Preziosi, L., Lo Buono, N., Funaro, A.: Individual cellbased model for in-vitro mesothelial invasion of ovarian cancer. Math. Model. Nat. Phenom. 5(1), 203-223 (2010)

17. Osborne, J.M.: Multiscale model of colorectal cancer using the cellular potts framework. Cancer Inform. 14(Suppl. 4), 83-93 (2015)

18. Rubenstein, B.M., Kaufman, L.J.: The role of extracellular matrix in glioma invasion: a cellular potts model approach. Biophys J. 95(12), 5661-5680 (2008) 